\title{
Initial viral load and the outcomes of SARS
}

\author{
Chung-Ming Chu, Leo L.M. Poon, Vincent C.C. Cheng, Kin-Sang Chan, Ivan F.N. Hung, \\ Maureen M.L. Wong, Kwok-Hung Chan, Wah-Shing Leung, Bone S.F. Tang, Veronica L. Chan, \\ Woon-Leung Ng, Tiong-Chee Sim, Ping-Wing Ng, Kin-Ip Law, Doris M.W. Tse, Joseph S.M. Peiris, \\ Kwok-Yung Yuen
}

ß See related articles pages 1342, 1353

\section{Abstract}

Background: Severe acute respiratory syndrome (SARS) is caused by a novel coronavirus. It may progress to respiratory failure, and a significant proportion of patients die. Preliminary data suggest that a high viral load of the SARS coronavirus is associated with adverse outcomes in the intensive care unit, but the relation of viral load to survival is unclear.

Methods: We prospectively studied an inception cohort of 133 patients with virologically confirmed SARS who were admitted to 2 general acute care hospitals in Hong Kong from Mar. 24 to May 4, 2003. The patients were followed until death or for a minimum of 90 days. We used Cox proportional hazard modelling to analyze potential predictors of survival recorded at the time of presentation, including viral load from nasopharyngeal specimens (measured by quantitative reverse transcriptase polymerase chain reaction [PCR] of the SARS-associated coronavirus)

Results: Thirty-two patients (24.1\%) met the criteria for acute respiratory distress syndrome, and 24 patients (18.0\%) died. The following baseline factors were independently associated with worse survival: older age (61-80 years) (adjusted hazard ratio [HR] 5.24, 95\% confidence interval [CI] 2.03-13.53), presence of an active comorbid condition (adjusted HR 3.36, 95\% CI 1.44-7.82) and higher initial viral load of SARS coronavirus, according to quantitative PCR of nasopharyngeal specimens (adjusted HR 1.21 per $\log _{10}$ increase in number of RNA copies per millilitre, 95\% Cl 1.06-1.39).

Interpretation: We found preliminary evidence that higher initial viral load is independently associated with worse prognosis in SARS. Mortality data for patients with SARS should be interpreted in light of age, comorbidity and viral load. These considerations will be important in future studies of SARS.

CMAJ 2004;171(11):1349-52

7 he cause of SARS has been documented as a novel coronavirus. ${ }^{1-5}$ The total number of cases worldwide has reached more than 8000 , and 774 deaths occurred between Nov. 1, 2002, and July 31, 2003.

The case-fatality ratio for SARS has varied from $7 \%$ to $17 \%$ in the most severely affected areas, ${ }^{6}$ but the reason for such variation has remained speculative. Several studies conducted early in the epidemic examined short-term adverse outcomes of SARS (admission to intensive care unit $[\mathrm{ICU}]$ or death).$^{7-11}$ Data on the ultimate survival of patients with SARS were not available from these early studies, and some studies were performed before the SARS coronavirus had been identified; moreover, the factors identified in these studies as being associated with survival were generally not modifiable. ${ }^{12}$

In a previous small-scale study (23 patients) ${ }^{13}$ a high plasma or serum concentration of the SARS coronavirus was correlated with the short-term adverse outcome of admission to the ICU; however, ultimate outcomes were not reported in that study. In another study, viral replication in the nasopharynx was associated with more severe disease and diarrhea, and the peak viral load was associated with mortality. ${ }^{14}$ However, the peak viral load can be a result of multiple factors, such as treatment received and host clearance, and it is not known if the initial viral load, before initiation of treatment, is related to outcome.

We prospectively studied factors predicting the development of acute respiratory distress syndrome (ARDS) and the ultimate survival of patients with virologically confirmed SARS. In particular, we examined the hypothesis that high initial viral load would be associated with poorer outcomes. These results could have significant implications in future studies of SARS.

\section{Methods}

In this prospective observational study, we examined the occurrence of ARDS or death in an inception cohort of adult patients with virologically confirmed SARS. We recruited 133 consecutive SARS patients admitted to the United Christian Hospital and the Caritas Medical Centre, Hong Kong, from Mar. 24 to May 4, 2003.

The subjects included in the study met a modified World Health Organization definition of SARS, ${ }^{8}$ with virologic confirmation of infection by the SARS coronavirus (paired serologic testing with or without positive result on reverse transcriptase polymerase chain reaction [PCR] for SARS coronavirus in clinical 
specimens). The paired serologic testing was performed on presentation and at day 28 after presentation. The virologic diagnostic protocol has been published previously. ${ }^{1,8}$ All patients were treated with broad-spectrum antibiotics, ribavirin and a tapering course of corticosteroid,$^{15}$ as detailed in our previous publication. ${ }^{8}$ The complete clinical and virologic features of the first 75 patients have been previously reported. ${ }^{8}$ The study was approved by the ethics committees of the United Christian Hospital and Caritas Medical Centre.

Patients who experienced respiratory failure were intubated and supported with mechanical ventilation, except if they or their surrogates refused intubation. Patients who received other forms of experimental treatment were excluded from the study.

Trained nurses collected nasopharyngeal specimens at the time of presentation, before any treatment. RNA was extracted by means of a QIAamp viral kit (QIAGEN GmbH, Hilden, Germany) according to the manufacturer's instructions. For the quantitative PCR (qPCR) assay, RNA and cDNA (complementary DNA) were generated as previously described..$^{16} \mathrm{cDNA}$ was amplified in a model 7000 sequence detection system (Applied Biosystems) with the TaqMan PCR core reagent kit (Applied Biosystems, Applera Corporation, Foster City, Calif.). To monitor the integrity of the RNA and to test whether these samples contained any PCR inhibitor, a real-time reverse transcriptase PCR for $\beta$-actin mRNA (messenger RNA) was also performed

\begin{tabular}{|c|c|c|c|}
\hline \multirow[b]{2}{*}{ Baseline variable } & \multicolumn{2}{|c|}{ Patient group; result, mean $(\mathrm{SD})^{*}$} & \multirow[b]{2}{*}{$p$ value } \\
\hline & $\begin{array}{c}\text { No ARDS } \\
n=101\end{array}$ & $\begin{array}{l}\text { ARDS } \\
n=32\end{array}$ & \\
\hline Age, yr & $37.5(11.8)$ & $46.0(14.2)$ & $0.001+$ \\
\hline Sex, \% male & $36(36)$ & $18(56)$ & $0.06 \neq$ \\
\hline $\begin{array}{l}\text { Active comorbid condition(s), no. (\%) } \\
\text { of patients }\end{array}$ & $10(10)$ & $11(34)$ & $0.002 \ddagger$ \\
\hline $\begin{array}{l}\text { Normal result on initial chest radiography, } \\
\text { no. }(\%) \text { of patients }\end{array}$ & $25(25)$ & $7(22)$ & $0.826 \neq$ \\
\hline $\begin{array}{l}\text { Multilobar involvement on presentation, } \\
\text { no. }(\%) \text { of patients }\end{array}$ & $17(17)$ & $10(31)$ & $0.08 \ddagger$ \\
\hline Temperature, ${ }^{\circ} \mathrm{C}$ & $38.8(1.2)$ & $38.9(0.9)$ & $0.58 \dagger$ \\
\hline Oxygen saturation, $\%$ & $97.9(1.2)$ & $97.5(1.6)$ & $0.19 \dagger$ \\
\hline Respiratory rate, breaths/min & $20.1(2.8)$ & $20.2(4.0)$ & $0.88 \dagger$ \\
\hline Pulse, beats/min & $101(16)$ & $101(19)$ & $0.94 \dagger$ \\
\hline Hemoglobin, g/dL & $13.4(1.5)$ & $13.3(1.8)$ & $0.77 \dagger$ \\
\hline Leukocyte count, $\times 10^{9} / \mathrm{L}$ & $6.1(2.1)$ & $6.5(2.9)$ & $0.86 \dagger$ \\
\hline Lymphocyte count, $\times 10^{9} / \mathrm{L}$ & $0.9(0.4)$ & $1.0(0.7)$ & $0.21 \dagger$ \\
\hline Platelet count, $\times 10^{9} / \mathrm{L}$ & $176(49)$ & $166(50)$ & $0.35 \dagger$ \\
\hline Urea level, mmol/L & $4.0(1.2)$ & $4.3(1.5)$ & $0.26 \dagger$ \\
\hline Creatinine level, $\mathrm{mmol} / \mathrm{L}$ & $84(16)$ & $88(17)$ & $0.24 \dagger$ \\
\hline $\begin{array}{l}\text { Lactate dehydrogenase level, median } \\
\text { (IQR), IU/L }\end{array}$ & $364(293-441)$ & $404(322-520)$ & $0.034 \S$ \\
\hline Viral load, $\uparrow$ median (IQR), $\log _{10}$ copies $/ \mathrm{mL}$ & $0(0-4.6)$ & $4.7(0-6.3)$ & $0.025 \S$ \\
\hline
\end{tabular}

Note: $\mathrm{SD}=$ standard deviation, $\mathrm{IQR}=$ interquartile range.

*Unless specified otherwise.

†Student's $t$ test.

Fisher's exact test.

§Mann-Whitney $U$ test.

I Determined by quantitative polymerase chain reaction of SARS coronavirus from nasopharyngeal specimen. (forward primer 5'-CCCAAGGCCAACCGCGAGAAGAT-3' and reverse primer 5'-GTCCCGGCCAGCCAGGTCCAG-3').

Patients were followed for a minimum of 90 days to track development of ARDS (defined as ratio of arterial oxygen pressure to fraction of inspired oxygen less than $200 \mathrm{~mm} \mathrm{Hg}^{17}$ ) and death. Data for patients who were still alive at the end of follow-up (Aug. 31, 2003) were censored.

All timed data were recorded from onset of SARS symptoms. Results are expressed as mean (and standard deviation [SD]) unless otherwise specified. Univariate analyses were used to compare baseline clinical, biochemical, virologic and radiographic data in patients with and without ARDS. Student's $t$ test and the Mann-Whitney $U$ test were used for continuous variables as appropriate; Fisher's exact test was used for categorical variables. Multiple logistic regression was used to assess baseline factors that might be associated with the development of ARDS. The Cox proportional hazards model was used to assess baseline factors that might be associated with poor survival; graphic and statistical checks for proportionality of hazards were performed. Factors significantly associated with death $(p<0.05)$ and known factors from previous studies were further examined by multivariate Cox regression to identify independent risk factors for reduced survival. The Kaplan-Meier product-limit estimator was used to estimate survival and for the time-to-death plot. Data are reported as the hazard ratio (HR) with $95 \%$ confidence interval (CI). A 2 -tailed $p$ value of less than 0.05 was considered statistically significant.

\section{Results}

Baseline and follow-up data were complete for the 133 cases. All surviving patients had been discharged at the time of writing, and the median follow-up time for these cases was 122 days.

All patients had virologic documentation of infection with the SARS coronavirus by paired serologic testing. The mean age was 39.5 (SD 12.9) years, and there were 54 men $(40.6 \%)$ and 79 women $(59.4 \%)$. Pretreatment nasopharyngeal specimens were obtained at a mean of 3.9 (SD 1.9) days after onset of symptoms. All nasopharyngeal samples collected contained detectable $\beta$-actin mRNA. The initial results of qPCR of the nasopharyngeal specimens ranged from undetectable to $8.8 \log _{10}$ RNA copies $/ \mathrm{mL}$. There was no correlation between the viral load and the number of days elapsed from symptom onset to presentation (Spearman's $\rho=-0.079, p=0.42$ ).

ARDS developed in 32 (24.1\%) of the 133 patients. Of these 32 patients, 26 (19.5\% of the total cohort) were put on ventilatory support, and 19 died $(14.3 \%$ of the total cohort). Six of the 32 patients with ARDS did not receive ventilation. 
For 5 of these (3.8\% of the total cohort), ventilatory support was withheld at the patient's or a surrogate's request, and these 5 patients died; for the sixth patient intubation was deferred and the patient subsequently made a full recovery without ventilation. There were a total of 24 deaths $(18.0 \%)$ in the cohort. All of the deceased patients had intractable hypoxemia due to ARDS at the time of death; 5 (21\%) of the 24 also had nosocomial sepsis and multiorgan failure, $2(8 \%)$ had acute myocardial infarction, 1 (4\%) had disseminated intravascular coagulopathy, and 1 (4\%) had end-stage renal failure.

\section{Predictive factors for ARDS}

On univariate analysis, the following baseline factors were associated with ARDS: age $(p=0.001)$, comorbidity $(p=0.002)$, initial lactate dehydrogenase level $(p=0.034)$ and $\mathrm{qPCR}$ result of the nasopharyngeal specimen $(p=$ 0.025 ) (Table 1). The crude odds ratio (OR) for qPCR of nasopharyngeal specimens (per $\log _{10}$ increase in number of RNA copies per millilitre) for the development of ARDS was 1.17 (95\% CI $1.02-1.34 ; p=0.03$ ).

Multiple logistic regression was used to examine whether the initial viral load was independently associated with ARDS; data on viral load were entered into models containing known prognostic factors (age, comorbidity and initial lactate dehydrogenase level). In only one model (together with comorbidity) was initial viral load independently associated with ARDS (adjusted OR 1.19, 95\% CI 1.03-1.38; $p=0.018$ ).

\section{Predictive factors for reduced survival}

On univariate analysis by the Cox proportional hazards model, the following baseline factors were associated with reduced survival: age (HR 1.06, 95\% CI 1.03-1.08), male sex (HR 3.3, 95\% CI 1.4-7.7), comorbidity (HR 3.53, 95\% CI 1.54-8.07), creatinine level (HR 1.03, 95\% CI 1.001.05), initial lactate dehydrogenase level (HR 1.002, 95\% CI 1.001-1.003) and qPCR result of the nasopharyngeal specimen (HR 1.20 per $\log _{10}$ increase in number of RNA copies per millilitre, 95\% CI 1.05-1.37).

Multivariate Cox regression showed that 3 factors were independently associated with reduced survival: older age (61-80 years) (adjusted HR 5.24, 95\% CI 2.03-13.53), co-

\begin{tabular}{lcc}
\hline \multicolumn{3}{l}{ Table 2: Independent risk factors predicting death } \\
\hline Risk factor (on presentation) & Adjusted HR $(95 \% \mathrm{Cl})$ & $p$ value \\
\hline Age, yr & & 0.002 \\
$21-40$ & 1.00 & \\
$41-50$ & $1.35(0.51-3.54)$ & 0.55 \\
$\quad 61-80$ & $5.24(2.03-13.53)$ & 0.001 \\
Active comorbid condition & $3.36(1.44-7.82)$ & 0.005 \\
Viral load, ${ }^{2}$ per log lincrease $_{\text {in RNA copies/mL }}$ & $1.21(1.06-1.39)$ & 0.006 \\
\hline
\end{tabular}

Note: $\mathrm{HR}=$ hazard ratio, $\mathrm{Cl}=$ confidence interval.

*Determined by quantitative polymerase chain reaction of SARS coronavirus from nasopharyngeal specimen. morbidity (adjusted HR 3.36, 95\% CI 1.44-7.82) and qPCR result of the nasopharyngeal specimen (adjusted HR 1.21 per $\log _{10}$ increase in number of RNA copies per millilitre, 95\% CI 1.06-1.39) (Table 2). A Kaplan-Meier plot with respect to the initial viral load is shown in Fig. 1.

\section{Interpretation}

SARS is associated with significant mortality, and in this study increasing age and presence of active comorbid condition(s) were associated with worse survival. We also found preliminary evidence that the initial (pretreatment) viral load, determined by qPCR of nasopharyngeal specimens, was independently associated with eventual mortality. In a previous study high plasma or serum concentrations of SARS coronavirus were related to the short-term adverse outcome of ICU admission. ${ }^{13}$ In another study, the peak nasopharyngeal viral load was associated with mortality. ${ }^{14}$ Results from the current study suggest that pretreatment viral load influenced the ultimate outcome in terms of death. Little is known about the early pathologic changes of SARS in the lungs, as most histopathologic studies have been conducted post mortem. ${ }^{18}$ Further research is needed to investigate the relation between viral load and lung abnormalities and how it might affect treatment.

It is difficult to compare case-fatality ratios between different cohorts and countries. Differences in distribution in terms of age, sex, disease activity (reflected by lactate dehydrogenase level) and proportion of cases confirmed by virologic tests have been cited as possible reasons for differences in case-fatality ratios. ${ }^{12}$ Our results suggest that such variation should be interpreted in light of host factors (age and

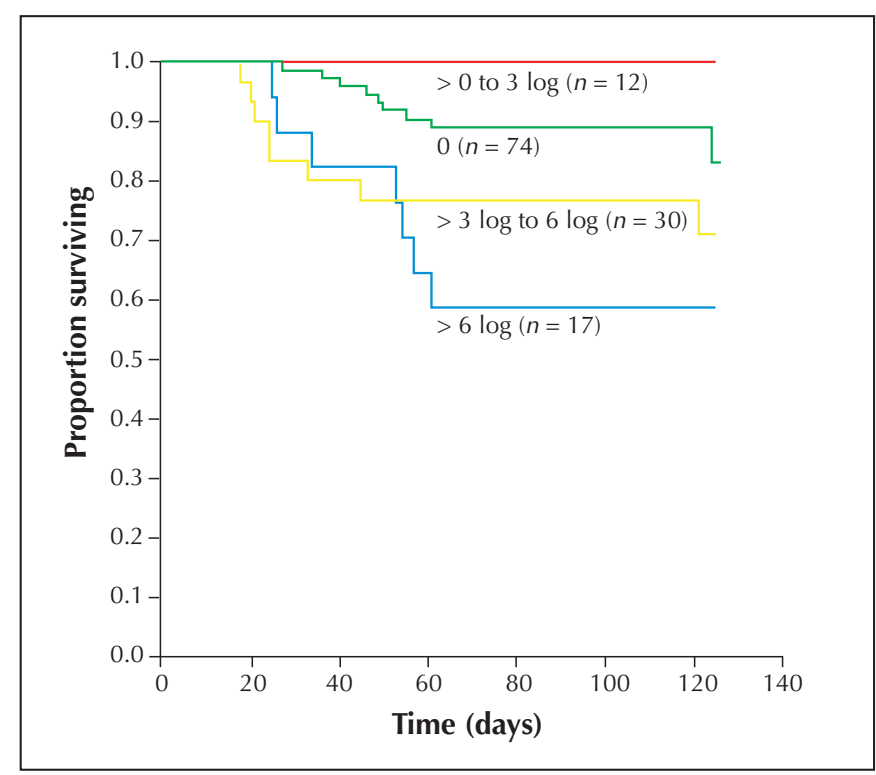

Fig 1: Kaplan-Meier survival curves according to level of SARS coronavirus (on the basis of number of RNA copies per millilitre), as determined by quantitative reverse transcriptase polymerase chain reaction of initial nasopharyngeal specimens. 
comorbidity) and viral factors (viral load). In future studies, viral load should be monitored and analyzed, so that it can be considered in the interpretation of outcome data.

Our study had some limitations. First, it was limited to 2 centres. It was not possible to perform a large-scale multicentre study during the epidemic, as qPCR for SARS coronavirus was available only in research laboratories at that time. However, the overall mortality rate in this study was similar to the estimated mortality rates in a large-scale study of 1425 cases, ${ }^{19}$ which suggests that our cohort was representative of typical SARS cases. Another limitation was the lack of a validation sample. Such a sample was not available because the SARS epidemic was brought under control quickly, once effective public health and hospital infection control measures were implemented. In addition, because we set out to explore a range of potential prognostic factors, the number of variables tested was large relative to the event rates. Our results should be regarded as preliminary because of the increased probability that they are due to chance (because of this multiple testing). Finally, we did not investigate whether viral load in other specimens (e.g., stool or serum) was an important determinant of clinical outcomes.

We have provided preliminary evidence that higher initial nasopharyngeal viral load is independently associated with worse prognosis in SARS. Mortality data for SARS should be interpreted in light of age, comorbidity and viral load. Monitoring of SARS coronavirus by qPCR should be an integral part of future SARS studies.

\section{This article has been peer reviewed.}

From the Department of Medicine and Geriatrics (Chu, K.S. Chan, Leung, V.L. Chan, W.L. Ng, Sim, P.W. Ng) and the Intensive Care Unit (Law), United Christian Hospital, Hong Kong Special Administrative Region, China; the Department of Microbiology, Queen Mary Hospital, The University of Hong Kong (Poon, Cheng, Hung, Tang, K.H. Chan, Peiris, Yuen), Hong Kong Special Administrative Region, China; and the Department of Medicine and Geriatrics, Caritas Medical Centre (Wong, Tse), Hong Kong Special Administrative Region, China

\section{Competing interests: None declared.}

Contributors: Conception and design: Chung-Ming Chu, Kin-Sang Chan, KwokYung Yuen; acquisition of clinical data: Chung-Ming Chu, Maureen Wong, WahShing Leung, Veronica Chan, Woon-Leung Ng, Tiong-Chee Sim, Ping-Wing Ng, Kin-Ip Law, Doris Tse; acquisition of virological data: Leo Poon, Vincent Cheng, Ivan Hung, Kwok-Hung Chan, Bone Tang, Joseph Peiris, Kwok-Yung Yuen; analysis and interpretation of data: Chung-Ming Chu, Leo Poon, Vincent Cheng, Kin-Sang Chan, Kwok-Yung Yuen; drafting of manuscript: Chung-Ming Chu, Leo Poon, Vincent Cheng, Kin-Sang Chan; and critical revision of manuscript for important intellectual content: Chung-Ming Chu, Kin-Sang Chan, Kwok-Yung Yuen. All authors critically reviewed and approved the final version to be published.

Acknowledgements: We acknowledge research funding from the Suen Chi Sun Charitable Foundation, the Hong Kong University SARS Donation Fund (DBS) and an RGC grant (HKU7543/03M).

\section{References}

1. Peiris JS, Lai ST, Poon LL, Guan Y, Lam LY, Lim W, et al. Coronavirus as a possible cause of severe acute respiratory syndrome. Lancet 2003;361:1319-25.

2. Ksiazek TG, Erdman D, Goldsmith CS, Zaki SR, Peret T, Emery S, et al. A novel coronavirus associated with severe acute respiratory syndrome. $N$ Engl 7 Med 2003;348:1953-66

3. Drosten C, Gunther S, Preiser W, van der Werf S, Brodt HR, Becker S, et al. Identification of a novel coronavirus in patients with severe acute respiratory syndrome. N Engl 7 Med 2003;348:1967-76.

4. Fouchier RA, Kuiken T, Schutten M, van Amerongen G, van Doornum GJ van den Hoogen BG, et al. Aetiology: Koch's postulates fulfilled for SARS virus. Nature 2003;423:240

5. Kuiken T, Fouchier RA, Schutten M, Rimmelzwaan GF, van Amerongen G van Riel D, et al. Newly discovered coronavirus as the primary cause of severe acute respiratory syndrome. Lancet 2003;362:263-70.

6. World Health Organization. Summary of probable SARS cases with onset of illness from 1 November 2002 to 31 July 2003. Geneva: The Organization; 2004. Available: www.who.int/csr/sars/country/table2004_04_21/en/ (accessed 2004 Oct 20)

7. Lee N, Hui D, Wu A, Chan P, Cameron P, Joynt GM, et al. A major outbreak of severe acute respiratory syndrome in Hong Kong. $N$ Engl $7 \mathrm{Med}$ 2003;348:1986-94.

8. Peiris JS, Chu CM, Cheng VC, Chan KS, Hung IF, Poon LL, et al. Clinical progression and viral load in a community outbreak of coronavirus-associated SARS pneumonia: a prospective study. Lancet 2003;361:1767-72.

9. Booth CM, Matukas LM, Tomlinson GA, Rachlis AR, Rose DB, Dwosh HA et al. Clinical features and short-term outcomes of 144 patients with SARS in the greater Toronto area. FAMA 2003;289:2801-9.

10. Wong RS, Wu A, To KF, Lee N, Lam CW, Wong CK, et al. Haematological manifestations in patients with severe acute respiratory syndrome: retrospective analysis. $B M \mathcal{F}$ 2003;326:1358-62.

11. Chan JW, Ng CK, Chan YH, Mok TY, Lee S, Chu SY, et al. Short term outcome and risk factors for adverse clinical outcomes in adults with severe acute respiratory syndrome (SARS). Thorax 2003;58:686-9.

12. Chan KS, Zheng JP, Mok YW, Li YM, Liu YN, Chu CM, et al. SARS: prognosis, outcome and sequelae. Respirology 2003;8(Suppl):S36-40.

13. Ng EK, Hui DS, Chan KC, Hung EC, Chiu RW, Lee N, et al. Quantitative analysis and prognostic implication of SARS coronavirus RNA in the plasma and serum of patients with severe acute respiratory syndrome. Clin Chem 2003;49:1976-80.

14. Cheng VC, Hung IF, Tang BS, Chu CM, Wong MM, Chan KH, et al. Vira replication in the nasopharynx is associated with diarrhea in patients with severe acute respiratory syndrome. Clin Infect Dis 2004;38:467-75.

15. So LK, Lau AC, Yam LY, Cheung TM, Poon E, Yung RW, et al. Development of a standard treatment protocol for severe acute respiratory syndrome. Lancet 2003;361:1615-7.

16. Poon LL, Wong OK, Chan KH, Luk W, Yuen KY, Peiris JS, et al. Rapid diagnosis of a coronavirus associated with severe acute respiratory syndrome (SARS). Clin Chem 2003;49:953-5.

17. Bernard GR, Artigas A, Brigham KL, Carlet J, Falke K, Hudson L, et al. The American-European consensus conference on ARDS: definitions, mechanisms, relevant outcomes, and clinical trial coordination. Am 7 Respir Crit Care Med 1994;149:818-24.

18. Nicholls JM, Poon LL, Lee KC, Ng WF, Lai ST, Leung CY, et al. Lung pathology of fatal severe acute respiratory syndrome. Lancet 2003;361:1773-8.

19. Donnelly CA, Ghani AC, Leung GM, Hedley AJ, Fraser C, Riley S, et al. Epidemiological determinants of spread of causal agent of severe acute respiratory syndrome in Hong Kong. Lancet 2003;361:1761-6.

Correspondence to: Dr. Kwok-Yung Yuen, Department of Microbiology, Queen Mary Hospital, The University of Hong Kong, Pokfulam Road, Hong Kong, SAR, China; fax 852 2855124; kyyuen@hkucc.hku.hk 